\title{
A Study on the Influence of Number/Distribution of Sensing Points of the Smart Insoles on the Center of Pressure Estimation for the Internet of Things Applications
}

\author{
Li-Wei Chou $1,2,3 \oplus$, Jun-Hong Shen ${ }^{4,5}$, Hui-Ting Lin ${ }^{6}$, Yi-Tung Yang ${ }^{3}$ and Wen-Pin $\mathrm{Hu}^{5,7, * \mathbb{C}}$ \\ 1 Department of Physical Medicine and Rehabilitation, China Medical University Hospital, \\ Taichung City 40402, Taiwan; chouliwe@gmail.com \\ 2 Department of Physical Therapy, Graduate Institute of Rehabilitation Science, China Medical University, \\ Taichung City 40402, Taiwan \\ 3 Department of Physical Medicine and Rehabilitation, Asia University Hospital, Taichung City 41354, Taiwan \\ t00031@auh.org.tw \\ 4 Department of Information Communication, Asia University, Taichung City 41354, Taiwan; \\ shenjh@asia.edu.tw \\ 5 Department of Medical Research, China Medical University Hospital, China Medical University, \\ Taichung City 40447, Taiwan \\ 6 Department of Physical Therapy, I-Shou University, Kaohsiung City 82445, Taiwan; huitinglin@isu.edu.tw \\ 7 Department of Bioinformatics and Medical Engineering, Asia University, Taichung City 41354, Taiwan \\ * Correspondence: wenpinhu@asia.edu.tw; Tel.: +886-4-23323456 (ext. 20022)
}

\section{check for} updates

Citation: Chou, L.-W.; Shen, J.-H.; Lin, H.-T.; Yang, Y.-T.; Hu, W.-P. A Study on the Influence of Number/Distribution of Sensing Points of the Smart Insoles on the Center of Pressure Estimation for the Internet of Things Applications. Sustainability 2021, 13, 2934. https:// doi.org/10.3390/su13052934

Academic Editor: Arun

Kumar Sangaiah

Received: 9 February 2021

Accepted: 5 March 2021

Published: 8 March 2021

Publisher's Note: MDPI stays neutral with regard to jurisdictional claims in published maps and institutional affiliations.

Copyright: (c) 2021 by the authors. Licensee MDPI, Basel, Switzerland. This article is an open access article distributed under the terms and conditions of the Creative Commons Attribution (CC BY) license (https:/ / creativecommons.org/licenses/by/ $4.0 /)$.

\begin{abstract}
The past decade has seen the emergence of numerous new wearable devices, including many that have been widely adopted by both physicians and consumers. In this paper, we discuss the design and application of smart insoles to measure gait and plantar pressure. Herein, we investigate the potential applications of insoles with fewer sensing spots and the consequent reduction in the amount of data acquired from both feet. The main purpose is to discuss the influence of the layout of these pressure sensing points of the insole design on the center of pressure (COP) calculation. The insole used in this study has 89 pressure sensing spots, and we used data from 36, 29, 20, and 11 sensing points in simplified calculation types. Among these four simplified calculation types, Type 1 exhibited the best accuracy of the COP calculation, and Type 4 obtained the worst results. Type 2 and Type 3 exhibited inferior accuracy of the COP calculation, but they still sufficed for applications that did not require high accuracy. Aside from the factor of the number of sensing spots used in the calculation, we also demonstrated that the location of selected sensors could influence the accuracy of COP calculation in the analyses by using the different combinations of metatarsal areas and other areas (heel, central, lateral toes, and hallux). The results of this research could be a reference for making a simplified form of pressure sensing Internet-of-Health Things (IoHT) insole with a reduced product cost.
\end{abstract}

Keywords: center of pressure (COP); Internet-of-Health Things (IoHT); pressure; sensing insole; foot; analysis

\section{Introduction}

The rapid evolution of the Internet of Things (IoT) has encouraged swift progress in the development of various devices, such as air quality monitors, wearables, and smart home devices, that provide real-time, reliable data that can be analyzed through IoT analytics to realize value. The IoT system is not limited to machine-to-machine communication, but expands to sensor-to-machine interactions. These sensors can be categorized as either wearable or non-wearable. The most popular wearable electronic-based product in the market is the smart-watch, which can detect body signals such as heart rate and blood pressure [1], and also provide other healthcare functions such as fall detection and emergency alert [2]. 
IoT devices are able to achieve the concept of home automation, and these technologies can have medical applications. Internet-of-Health Things (IoHT), which are also known as Internet-of-Medical Things (IoMT), integrate the IoT and healthcare environments to record or monitor activities of the user for medical or healthcare purposes. Smart insoles are one of the wearable devices undergoing fast growth because smart insoles can acquire plantar pressure measurements and calculate gait information (step count, duration of the gait cycle, swing time, stance time, center of pressure (COP) velocity, etc.) [3]. This valuable information is expected to enable the detection of the early signs and symptoms of diseases that cause gait dysfunction, such as Alzheimer's disease [4], non-Alzheimer's dementia [5] and Parkinson's disease [6]. Plantar pressure information has also been proven to be valuable in the assessment of patients with diabetes $[7,8]$, medical knee osteoarthritis $[9,10]$ and peripheral neuropathy $[8,11]$. The foot pressure pattern in individuals with medical knee osteoarthritis is characterized by calculating partial foot pressure as a percentage of body weight (\%PFP), percentage of the transverse width of the COP path to the maximum foot width (\%Trans) and other simple parameters $[9,10]$. The butterfly diagram is a typical shape of the COP trajectory of an individual during walking, and can indicate gait characteristics [12]. Therefore, the accuracy of COP calculation is important in the assessment of the early signs and symptoms of diseases, including neurological and cerebellar impairment.

Center of pressure (COP) is commonly used for quantitative assessment of balance and gait, and the estimation of COP is usually measured by using force plates or expensive insole-based systems. Tekscan F-Scan [13] and Pedar [14] systems have been applied in numerous academic and clinical investigations, including diagnosing disease, rehabilitation assessment, sports biomechanics, gait analysis, and footwear research and design. However, these professional devices are expensive and consequently some low-cost insoles have been developed [15-18]. Lou, et al. [16] designed wearable pressure insoles to perform balance experiments, with each insole containing 28 sensing spots. They found the coefficients of variation of COP in the $x$ - and $y$-directions were similar between the insoles and Tekscan while calculating the distribution of COP in a standing posture (0.019 vs. 0.020 (Tekscan) in the x-direction; 0.016 vs. 0.021 (Tekscan) in the y-direction). The individual-specific nonlinear model used with the low-cost instrumented insoles (12 sensors) has demonstrated that it can improve the accuracy of foot plantar COP trajectory estimation [15]. Footwearbased wearable devices have great potential in commercial and clinical applications, and so some companies have developed and sold a variety of smart insoles, like Salted Smart insoles, Nuurv Run, Stridalyzer Insight Smart Insoles, etc. These insoles can connect to a mobile phone, or watch, and provide functions such as pressure distribution, foot motion tracking, and foot posture capturing.

A basic IoT model with edge processing for using smart insoles should incorporate the insoles, a device that can wirelessly transmit data, an edge computing platform, and a cloud network, as illustrated in Figure 1. The integration of IoT in healthcare can be termed IoHT. A real-time data calculation could provide more potential applications of smart insoles in rehabilitation medicine, sports injury detection, athlete training, timely rescue, and so on [19]. The mobile phone is a very suitable candidate for a receiver as it can perform calculations and transmit data to the edge computing platform. The data can also be transmitted through an indoor smart device. The information can then be immediately sent via the edge computing platform to the users, and their healthcare providers can also view the information from the cloud network. By using the technique of edge computing, the calculation workload of the mobile phone can be reduced, achieving the computation offloading in the IoT network [20]. The synchronization between wireless sensor nodes in the proposed model can be improved by using the multi-unit synchronized system for data logging [21]. In addition, due to the sensitivity of personal health data, privacy protection in electronic medical applications is a serious concern. The IoT networks used in healthcare environments have unique challenges and security requirements (integrity, authentication, privacy, and availability). 
Many different types of insoles have been developed to meet different needs, but few articles discuss the influence of the layout of these pressure sensing points on the COP calculation. A previous study explored the sensing cell configuration for the plantar pressure insole and found that the configuration of seven pressure sensing cells had the best COP trajectory that agreed well with the one obtained with the Tekscan system [22]. The aim of the study reported here is to investigate the influence of the number/distribution of these pressure sensing points of the insole design on the COP calculation. In order to investigate the effect on COP accuracy of a reduced data set, we proposed four types of simplified calculations using the sensing data. The results of COP trajectories calculated by using all 89 pressure sensing spots on the insole were used as the standard, and foot plantar $\mathrm{COP}$ and COP of a body were all analyzed. The influence of using different combinations of metatarsal areas and other areas (heel, central, lateral toes, and hallux) on the COP calculation was also studied. Accuracy comparisons were made between simplified and original types according to the average root mean square error (RMSE) of the COP trajectory. The contributions of this paper are summarized as follows:

1. We propose four simplified calculation types for the quantification of influence on the accuracy of COP calculation, which is valuable for the development of low-cost insole.

2. Results indicate that the design of the low-cost smart insole must consider the distribution of sensing spots.

3. We recommend that the number of sensing spots on the insole needs to be equal or greater than 20 in accordance with study results.

We structure the paper as follows: Section 1 describes the background and previous works on the development of smart insoles and IoT as well as the rationale for this study. After that, the methodology used in this research is described in Section 2. Section 3 presents the experimental results, and then Section 4 discusses the findings in this study. Finally, Section 5 provides some concluding remarks.

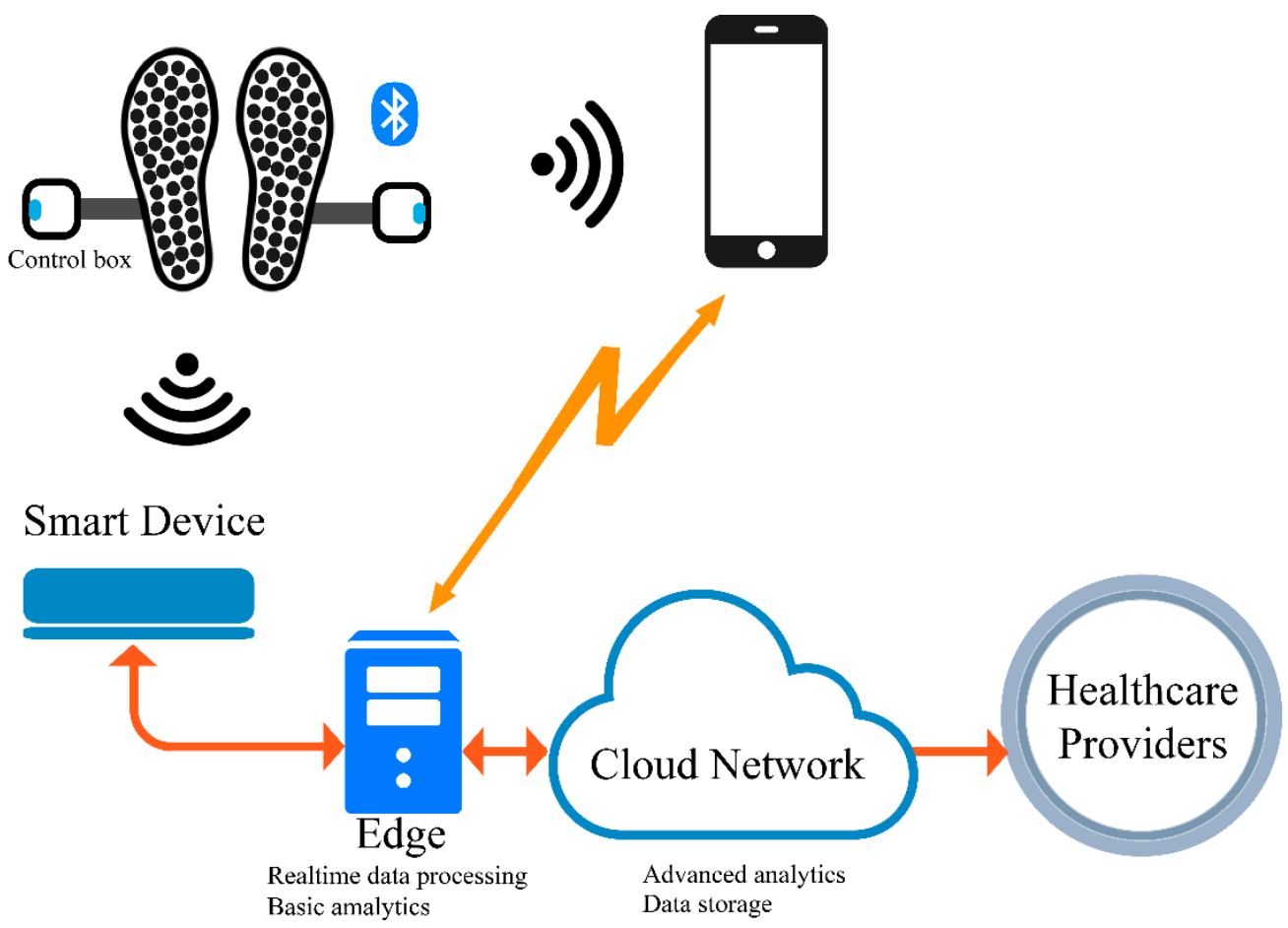

Figure 1. A general model for an IoT system with the edge computing for pressure sensing insoles.

\section{Materials and Methods}

A total of four subsections are presented in Materials and Methods. Section 2.1 describes the specifications and appearance of the smart insoles, and Section 2.2 illustrates 
the experimental procedures for the acquirement of data. We present the equations for the COP estimations, several configurations used for the data analysis and the statistical method in Section 2.3. The original configuration (using the data from all sensing spots) will be the reference for the calculation of displacements of the other 4 simplified configurations (using fewer sensing data). A general idea of the experimental design in this study is to propose several simplified configurations based on the original configuration of the pressure-sensing insole with 89 spots for the evaluation of the accuracy of COP estimation of proposed configurations. In the last subsection, the method for studying the influence of the locations of selected sensors illustrates here.

\subsection{Smart Insole}

The insole used in this study was developed by Industrial Technology Research Institute (Hsinchu County, Taiwan). The layout of the piezoelectric insole device is shown in Figure 2; it contains 89 sensing spots which are screen printed and cured on to a PET $\left(\right.$ Mylar $\left.^{\mathrm{TM}}\right)$ film, with the diameter of each spot size being $10 \mathrm{~mm}$ and a resulting spatial resolution per spot of $1 \mathrm{~mm} \times 1 \mathrm{~mm}$ (detail specifications shown in Table S1). These sensing spots are uniformly distributed over all regions of the foot, including important pressure-sensing locations, such as the big toe, metatarsophalangeal joints, arch, and heel. The sensing insole can fit a maximum shoe size of 10 (US units) and can be trimmed to match the user's shoe size. All signals detected by the sensors are sent to a control box containing a micro-computer, a Bluetooth module, an ADC module and a lithium battery. The data measured by the insoles from both feet are transmitted wirelessly and recorded simultaneously on a laptop computer through Bluetooth. The program used in the laptop to receive the data was written using LabVIEW. The sampling frequency of the insole system was set at $30 \mathrm{~Hz}$, and the data transmitted from 89 sensing spots were recorded every $0.33 \mathrm{~s}$. In order to calculate the foot plantar COP, an insole coordinate system was adopted, with the origin of the coordinate system being defined as the intersection between the $x$-axis and the $y$-axis (shown in Figure 3). The $x$-axis is the line tangential to the bottom of the insole and the $y$-axis is about $4.3 \mathrm{~cm}$ away from the line tangential to the right edge of the insole of the left foot. Therefore, each sensing point in the system has a coordinate value, with the units being $\mathrm{cm}$. Figure 3 lists the coordinate values of the first 35 sensing points.

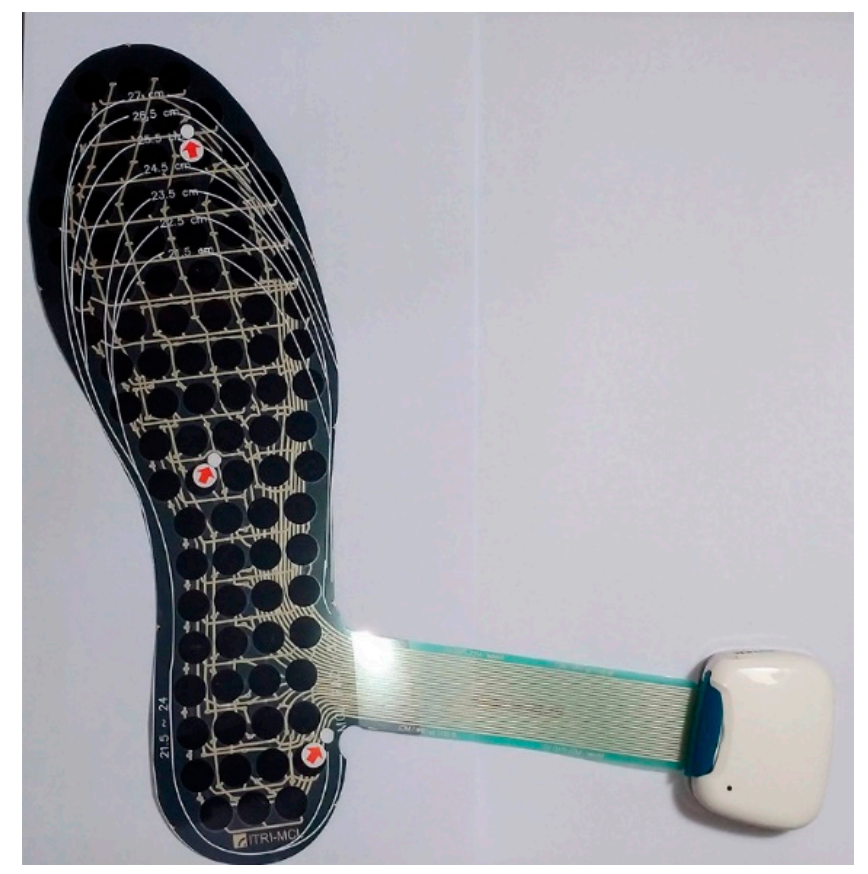

Figure 2. The layout of the pressure-sensing insole containing 89 sensing spots and developed by the Industrial Technology Research Institute (Taiwan). 
(5) lateral

(2) lateral toes

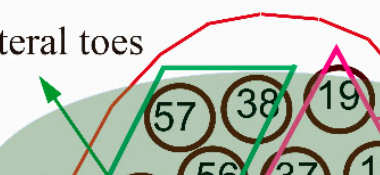
metata

(6) central<smiles></smiles>

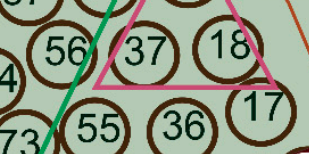

(1) hallux

(3) medial metatarsal
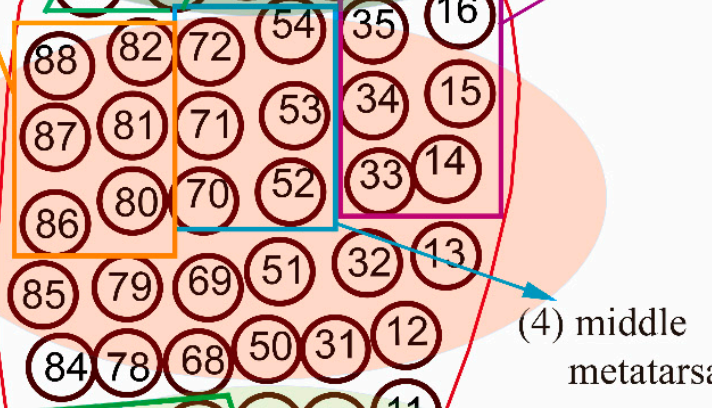

(4) middle metatarsal
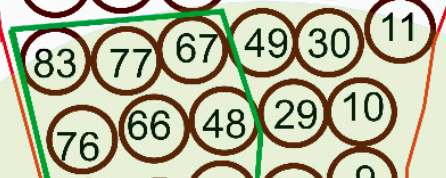

75) 65 (47) 28
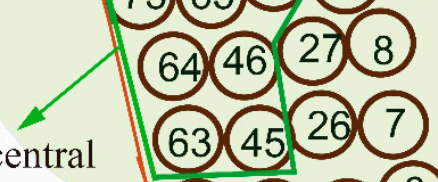

6244256

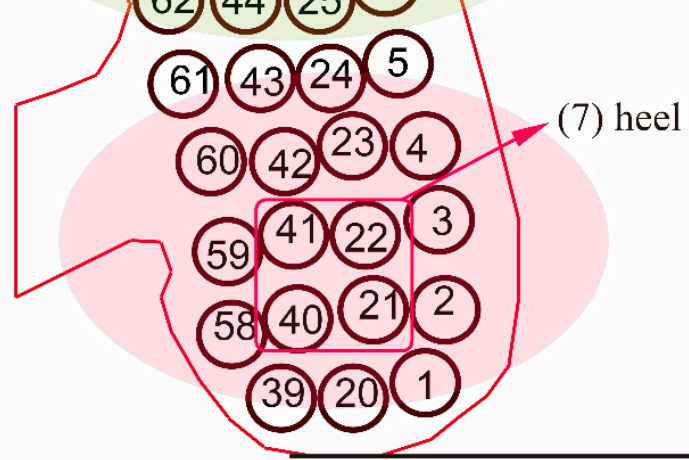

7) heel

\section{4}

\begin{tabular}{|c|c|c|}
\hline No. & $\mathrm{X}(\mathrm{cm})$ & $\mathrm{Y}(\mathrm{cm})$ \\
\hline 1 & -5.4 & 2.2 \\
\hline 2 & -5 & 3.5 \\
\hline 3 & -5.2 & 4.9 \\
\hline
\end{tabular}

\begin{tabular}{|l|l|l|}
\hline 3 & -5.2 & 4.9 \\
\hline 4 & -5.4 & 5.4 \\
\hline & -5.9 & \\
\hline
\end{tabular}

\begin{tabular}{|c|c|c|}
\hline 5 & -5.9 & 7.6 \\
\hline 6 & -6.2 & 9 \\
\hline
\end{tabular}

\begin{tabular}{|l|l|l|}
\hline 7 & -6.4 & 10.4 \\
\hline 8 & -6.1 & 11.4 \\
\hline 9 & -6.1 & 13.2 \\
\hline
\end{tabular}

\begin{tabular}{l|l|l|}
\hline 9 & -6.1 & 13.2 \\
\hline & -6.1 & 14.6 \\
\hline
\end{tabular}

\begin{tabular}{|l|l|l|}
\hline 10 & -6.1 & 14.6 \\
\hline 11 & -5.4 & 16.1 \\
\hline
\end{tabular}

\begin{tabular}{c|c|c}
12 & -5.5 & 17.5 \\
\hline 13 & -4.7 & 19
\end{tabular}

\begin{tabular}{|l|l|l}
\hline 14 & -4.7 & 20.3 \\
\hline 15 & -4.7 & 21.7 \\
\hline
\end{tabular}

\begin{tabular}{|l|l|l|}
\hline 16 & -4.7 & 23.2 \\
\hline 17 & -5.4 & 24.5 \\
\hline
\end{tabular}

\begin{tabular}{l|l|l}
17 & -5.4 & 24.5 \\
\hline 18 & -6.1 & 25.8 \\
\hline
\end{tabular}

\begin{tabular}{|c|c|c|}
\hline 19 & -6.9 & 27.1 \\
\hline 20 & -6.6 & 2.1 \\
\hline
\end{tabular}

\begin{tabular}{|l|l|l}
\hline 21 & -6.2 & 3.4 \\
\hline 22 & -6.4 & 4.8 \\
\hline
\end{tabular}

\begin{tabular}{|l|l|l|}
\hline 23 & -6.7 & 6.1 \\
\hline 24 & -7.1 & 7.5 \\
\hline
\end{tabular}

\begin{tabular}{l|l|r}
25 & -7.4 & 8.9 \\
\hline 26 & -7.6 & 10.2 \\
\hline
\end{tabular}

\begin{tabular}{|l|l|l|}
\hline 26 & -7.6 & 10.2 \\
\hline 27 & -7.9 & 11.7 \\
\hline 28 & -7.3 & 13.1 \\
\hline 29 & -7.3 & 14.5 \\
\hline
\end{tabular}

\begin{tabular}{l|l|l}
\hline 29 & -7.3 & 14.5 \\
\hline 30 & -6.7 & \\
\hline
\end{tabular}

\begin{tabular}{c|c|c}
30 & -6.7 & 16 \\
\hline 31 & -6.7 & 17.4
\end{tabular}

\begin{tabular}{|c|c|c|}
\hline 32 & -6.2 & 18.8 \\
\hline 33 & -6.1 & 20.2 \\
\hline 34 & -6.1 & 21.6 \\
\hline 35 & -6.1 & 23 \\
\hline
\end{tabular}

$x$

Figure 3. The pressure-sensing insole coordinate system. Only the coordinate values of the first 35 spots are listed here. The $\mathrm{x}$-coordinate values for the left foot are negative, and the $\mathrm{x}$-coordinate values of the right foot are positive. In the y-direction, the values are all positive for both feet. The spots that are selected for calculation during walking are marked as: heel, central, metatarsals, lateral toes, and hallux. The colored ellipses represent the four main regions of foot-printed area, including hallux and lateral toes, metatarsal, central, and heel (from top to bottom).

\subsection{The Experimental Design}

A total of seven healthy young male adults were recruited for the experiment (average age $=21.6 \pm 1.5$ years; height $=174.1 \pm 3.6 \mathrm{~cm}$; mass $=68.7 \pm 3.8 \mathrm{~kg}$ ), who had no musculoskeletal deficits or neurological impairment, nor had undergone lower extremity surgery. These participants all had the same shoe size of US 9.5, and their flat shoes were the same type and had the same sole. They all received and signed an informed consent form before data collection. The study protocol was approved by the China Medical University \& Hospital Research Ethics Committee, Taichung City, Taiwan. In this study, the participants were asked to perform two activities: (1) quiet standing with eyes open for $1 \mathrm{~min}$, and 
(2) standing up from a chair without armrests and walking $8 \mathrm{~m}$. In order to analyze the static motion of sitting and dynamic motion of walking, the data of sitting were retrieved from the initial section of data recorded from the second activity; the data for walking analysis were acquired starting from the time point that the subjects stood upright from the chair. We did not analyze the data recorded at the sit-to-stand stage. While sitting on the chair without armrests, the participants were asked to keep their feet on the ground before the action of standing up from the chair. The pressure-sensing insoles were inserted and put above the original insoles of the shoes by the experimenters. To avoid slippage between insoles, the pressure-sensing insoles were secured with double-sided tape. The participants were asked to wear the shoes prepared by the experimenters, and the boxes containing the electrical components were fixed firmly to the sides of the shoes. Before collecting the data, these participants had five minute's practice to become familiar with the feeling of wearing the prepared shoes. The data for each activity were recorded three times.

\subsection{Data Analysis for the COP Estimations and the Statistical Method}

The pressure data acquired from insoles were analyzed using Matlab programs (MathWorks, Inc., Natick, MA, USA). The COP trajectories of both feet were calculated, and the weighted-mean approach was applied to estimate the foot plantar COP trajectories. The foot plantar COP in the medial-lateral $(\mathrm{ML})$ direction $\left(C O P_{p x}\right)$ and the COP in the anteriorposterior $(\mathrm{AP})$ direction $\left(C O P_{p y}\right)$ were calculated according to Equations (1) and (2).

$$
\begin{aligned}
& C O P_{p x}=\frac{\sum C_{i}^{x} F_{i}}{F_{\text {total }}} \\
& C O P_{p y}=\frac{\sum C_{i}^{y} F_{i}}{F_{\text {total }}}
\end{aligned}
$$

where $C_{i}^{x}$ and $C_{i}^{y}$ represent the corresponding $\mathrm{x}$ and $\mathrm{y}$-axes locations of sensing spots defined in the coordinate system, respectively. $F_{i}$ is the value of pressure sensing spot, and $F_{\text {total }}$ is the sum of the values of sensing spots on the insole.

The center-of-pressure trajectory of a person in the medial-lateral $\left(C O P_{x}\right)$ and in the anterior-posterior $\left(C O P_{y}\right)$ directions were calculated by using Equations (3) and (4).

$$
\begin{aligned}
& C O P_{x}=\frac{C O P_{R, p x} \times F_{R, \text { total }}+C O P_{L, p x} \times F_{L, \text { total }}}{F_{R, \text { total }}+F_{L, \text { total }}} \\
& C O P_{y}=\frac{C O P_{R, p y} \times F_{R, \text { total }}+C O P_{L, p y} \times F_{L, \text { total }}}{F_{R, \text { total }}+F_{L, \text { total }}}
\end{aligned}
$$

where $C O P_{R, p x}$ and $C O P_{L, p x}$ represent the coordinate values of COP in the medial-lateral direction from the insoles of right and left feet, respectively. $C O P_{R, p y}$ and $C O P_{L, p y}$ are the coordinate values of COP in the anterior-posterior direction obtained from the two insoles on both feet. $F_{R, t o t a l}$ is the sum of all values measured by registering spots on the insole of the right foot, and $F_{L, t o t a l}$ is used to denote the sum from the left foot. The COP estimation is calculated by using the weighted-mean approach.

It is known that the accuracy of COP estimation depends on the areas covered by the pressure sensors while using the weighted-mean approach [15]. However, the error values of COP estimation in different configurations are not ever specifically quantified. To compare the effect caused by sensing spots on the accuracy of COP estimation, different numbers and arrangement types of sensing spots were adopted (shown in Figure 4a-e) to calculate the center-of-pressure trajectory. Figure 4 a shows the original type, containing 89 sensing spots. Type 1 (Figure $4 \mathrm{~b}$ ) uses 36 spots for the calculations. For Type 2, Type 3 and Type 4 (Figure $4 \mathrm{c}-\mathrm{e}$ ), the number of sensing spots used for the calculations of foot plantar COP and center-of-pressure trajectories are 29, 20 and 11, respectively. The data of 89 sensors were recorded at the same time in the experiments, and then we acquired the required data for calculations from the intact records according to the simplified 
configurations. The sensing configuration for Type 2 was developed based on Type 1 , with the only rule being that the selected sensing spots should be distributed as evenly as possible on the insole. For the sensing configurations of Type 3 and Type 4, we referenced the foot-print area during walking, including the heel, central area, metatarsals, lateral toes, and hallux [9], and also consulted the distribution of sensing spots of commercial pressure-sensing smart insole.

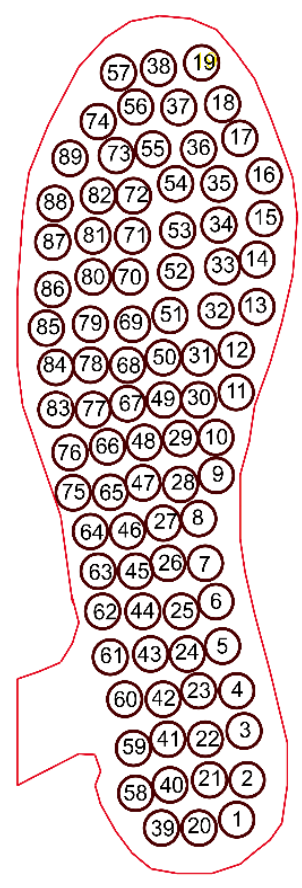

(a)

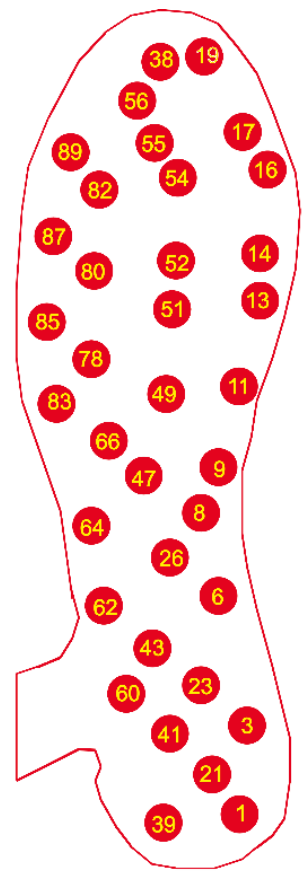

(b)

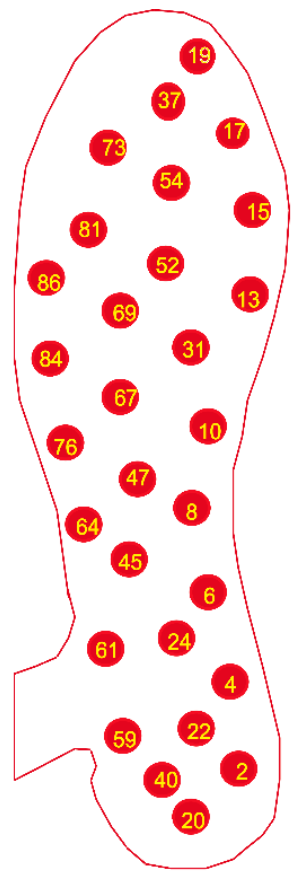

(c)

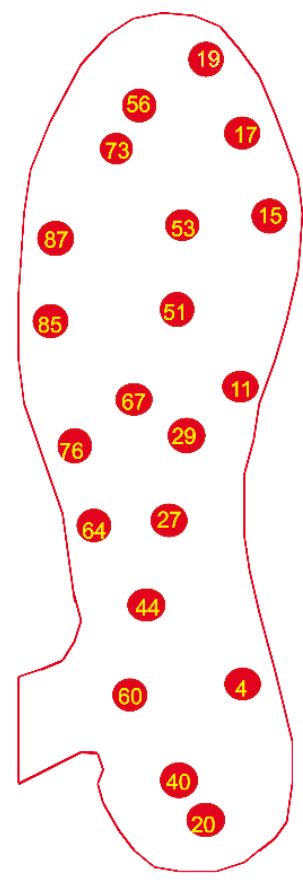

(d)

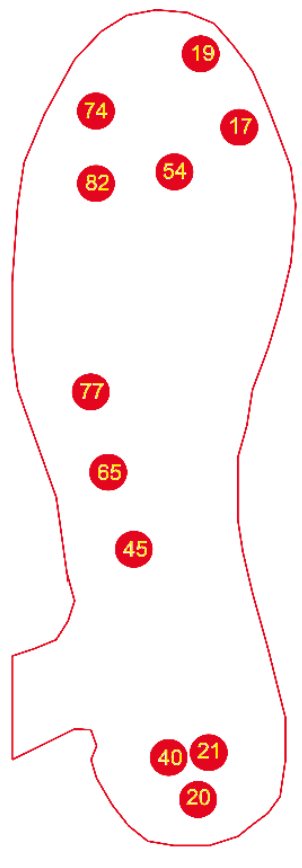

(e)

Figure 4. Different numbers of sensing spots used in the calculations according to the arrangement types (a) Original: 89 spots (b) Type 1: 36 spots (c) Type 2: 29 spots (d) Type 3: 20 spots (e) Type 4: 11 spots.

Based on the number of sensing spots, the original type should give the most accurate results of COP calculation. The data for the COP trajectory calculated according to the original type were used as the standard for comparison with the data obtained by adopting other arrangement. We used the difference distance of COP and RMSE (root mean square error) as the values for the quantitative comparison. The displacement of COP was calculated by the difference between the calculated positions of the original and other types of insoles. The displacement of COP was calculated according to Equations (5)-(7), where LocationD represents the difference of coordination values calculated from two calculation forms at the same time. RMSE was adopted as a measure of the differences between values obtained by the original type and the values calculated from other types. The lower the value of RMSE, the higher the accuracy.

$$
\begin{aligned}
& \text { Location }_{\mathrm{ML}, \mathrm{COP}}=\mathrm{X}_{\text {simplified type, } \mathrm{COP}}(\mathrm{t})-\mathrm{X}_{\text {original type, } \mathrm{COP}}(\mathrm{t}) \\
& \text { LocationD }_{\mathrm{AP}, \mathrm{COP}}=\mathrm{Y}_{\text {simplified type, } \mathrm{COP}}(\mathrm{t})-\mathrm{Y}_{\text {original type, } \mathrm{COP}}(\mathrm{t}) \\
& \text { Displacement }_{\mathrm{COP}}=\left(\text { LocationD }_{\mathrm{ML}, \mathrm{COP}}{ }^{2}+\text { Location }_{\mathrm{ML}, \mathrm{COP}^{2}}\right)^{1 / 2}
\end{aligned}
$$

We adopted the SPSS statistical software (version 18.0) to execute the statistical analysis. The data were examined using one-way ANOVA with the post-hoc Tukey test to test the differences between different groups. The cut-off values for $\mathrm{p}$ were set at $p<0.05$, $p<0.01$ and $p<0.001$, which are denoted $b^{*}{ }^{* *}$, and ${ }^{* * *}$, respectively. The $\mathrm{p}$ value has always been used as an important indicator to judge whether it is statistically significant. A statistically significant result indicates that the differences observed in this analysis. 
The G*Power software was utilized to calculate the statistical power for validating the test results.

\subsection{The Influence of the Locations of Selected Sensors}

In order to investigate the influence of the locations of selected sensors, we utilized another way to select the data from the sensing spots for calculation, with the spots being selected according to the foot-print area during walking; this foot-print area included the heel, central area, metatarsal area, lateral toes, and hallux. The selected spots included in these parts are shown in Figure 3. We sub-divided the metatarsal area into three regions: medial metatarsals, middle metatarsals, and lateral metatarsals. The spots in the heel, central area, lateral toes, and hallux are indispensable in these analyses, and changes in condition result from the combinations of different sub-parts of the metatarsal area. Therefore, we had four combinations with which to investigate the influence of the locations of selected sensors in the metatarsal area: (A) the complete metatarsal area; (B) medial and lateral metatarsal regions; (C) medial and middle metatarsal regions; (D) lateral and middle metatarsal regions. For the convenience of description, these four combinations were named A, B, C, and D, respectively. The number of sensing spots adopted in the A combination is 44 , and the number of sensing spots in other combinations is 38 .

\section{Results}

\subsection{Quiee Standing}

Figure 5 shows the COP trajectory estimation results acquired from subjects while quiet standing quietly. The COPx is plotted against COPy in Figure 5a, which shows the subject stable while during standing quietly, with the movement ranges in the $\mathrm{x}$ and $\mathrm{y}$ directions within 5 and $10 \mathrm{~mm}$, respectively. Figure $5 \mathrm{~b}-\mathrm{d}$ show the relationship between the displacement of $\mathrm{COP}$ and the number of data points. The displacement of COP indicates the straight line distance (2D displacement and be positive value) between the COP values calculated by using Type 1 and another Type. These figures represent different subjects who exhibit a similar trend. For subject 6 (shown in Figure 5d), his foot arch belongs to low arch. When using fewer sensing spots for the calculation (Type 4), the pressure distributed around the medial region of midfoot cannot be used for calculation. This factor causes quite a large change in the value of the displacement of COP.

Table 1 summarizes the RMSE values of COP trajectories during quiet standing; mean and standard deviation are presented in this table. Compared with the original type, Type 1 (with 36 sensing spots for the calculation) had the lowest RMSE values in the ML and AP directions. This consequence was reasonable because Type 1 used the largest amount of sensing data in the COP estimation among the four simplified calculation forms. For ML COPs of Type 2 and Type 3, the mean RMSE of Type 2 was smaller than that of Type 3. Nevertheless, in the AP direction, the mean RMSE of Type 2 COP reached a value of $11.3 \mathrm{~mm}$, which could be the cause of the larger displacement of COP shown in Figure 5. In addition, statistical results showed that the RMSE values for Type 1, Type 2, and Type 3 in the ML and AP directions were not significantly different. In the ML direction, there was only a significant difference between Type 2 and Type $4(p<0.05)$. There were very significant differences between Type 4 and Type 1 and Type $3(p<0.01)$, and a significant difference from Type $2(p<0.05)$.

Table 1. The average and standard deviation values of RMSE for different calculation types during quiet standing (values in table: mean \pm standard deviation; unit: $\mathrm{mm}$ ).

\begin{tabular}{ccccc}
\hline Types & Type 1 & Type 2 & Type 3 & Type 4 \\
\hline Direction & $1.8 \pm 1.0$ & $1.2 \pm 0.6$ & $5.5 \pm 3.3$ & $7.3 \pm 8.5$ \\
\hline ML direction & $6.0 \pm 2.1$ & $11.3 \pm 4.3$ & $6.3 \pm 6.6$ & $23.1 \pm 5.4$ \\
\hline AP direction & & & &
\end{tabular}




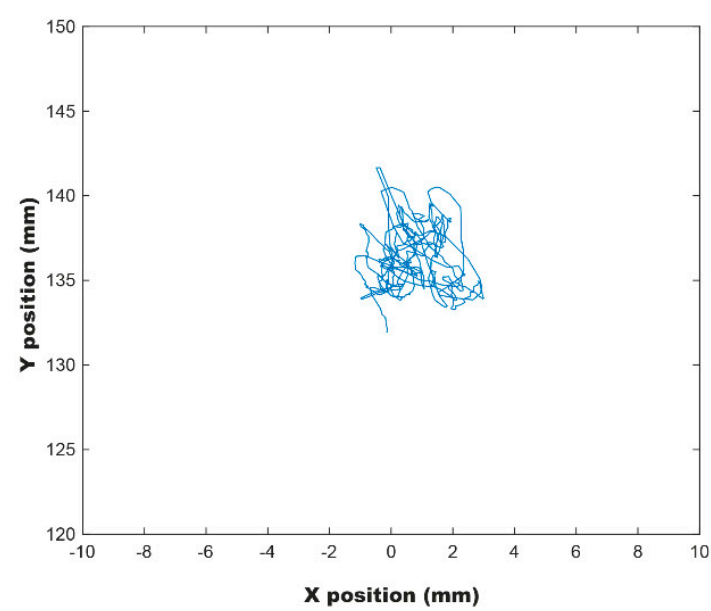

(a)

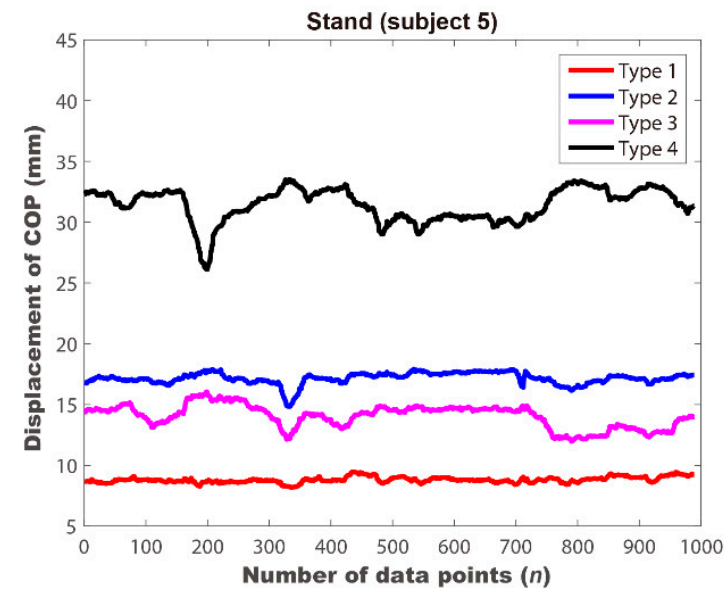

(c)

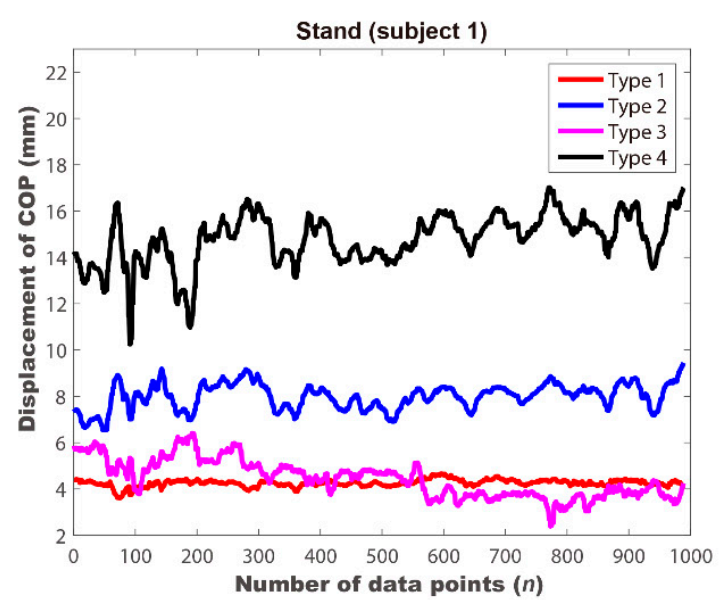

(b)

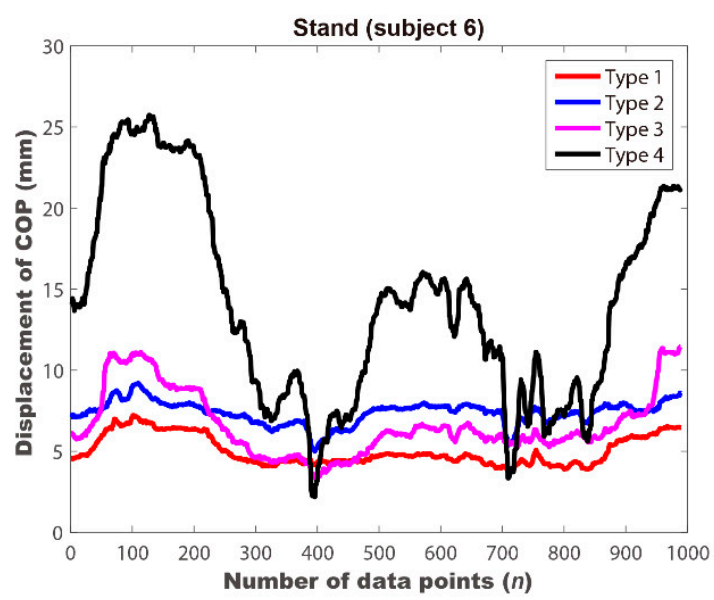

(d)

Figure 5. The trajectory and displacement of COP during quiet standing. (a) The representative center-of-pressure trajectory of a subject (subject 2) during quiet standing. The differences in the COP calculation by adopting different numbers of sensing spots for (b) subject 1 , (c) subject 5 and (d) subject 6 . The number of data points $(n)$ indicates the data collected at consecutive points in time.

\subsection{Sitting on Chair}

We retrieved and analyzed the static motion of sitting and motion of walking separately from "standing up from a chair and walking". It can be seen from Figure 6 that the deviation of COP estimation already exists in each type of calculation during sitting on the chair. Using fewer sensing spots in the calculation could lead to a larger deviation of COP estimation. Table 2 shows the RMSE values of COP trajectories during sitting on the chair without armrests. In this condition, the mass of the body mainly falls on the chair, and the loading conditions on the insoles are different from other motions. The average RMSE values in the ML and AP directions are very close for Type 1 and Type 2 . However, the deviations in the ML and AP directions become greater when the number of selected spots used in the COP calculation is equal to, or less than, 20. 
Table 2. The average and standard deviation values of RMSE for different calculation types during sitting on a chair without armrests (values in table: mean \pm standard deviation; unit: $\mathrm{mm}$ ).

\begin{tabular}{ccccc}
\hline Types & Type 1 & Type 2 & Type 3 & Type 4 \\
\hline Direction & $6.2 \pm 2.8$ & $5.1 \pm 2.8$ & $14.2 \pm 1.9$ & $10.1 \pm 9.0$ \\
\hline ML direction & $11.0 \pm 5.2$ & $11.0 \pm 3.5$ & $12.7 \pm 9.8$ & $25.9 \pm 10.8$ \\
\hline AP direction &
\end{tabular}

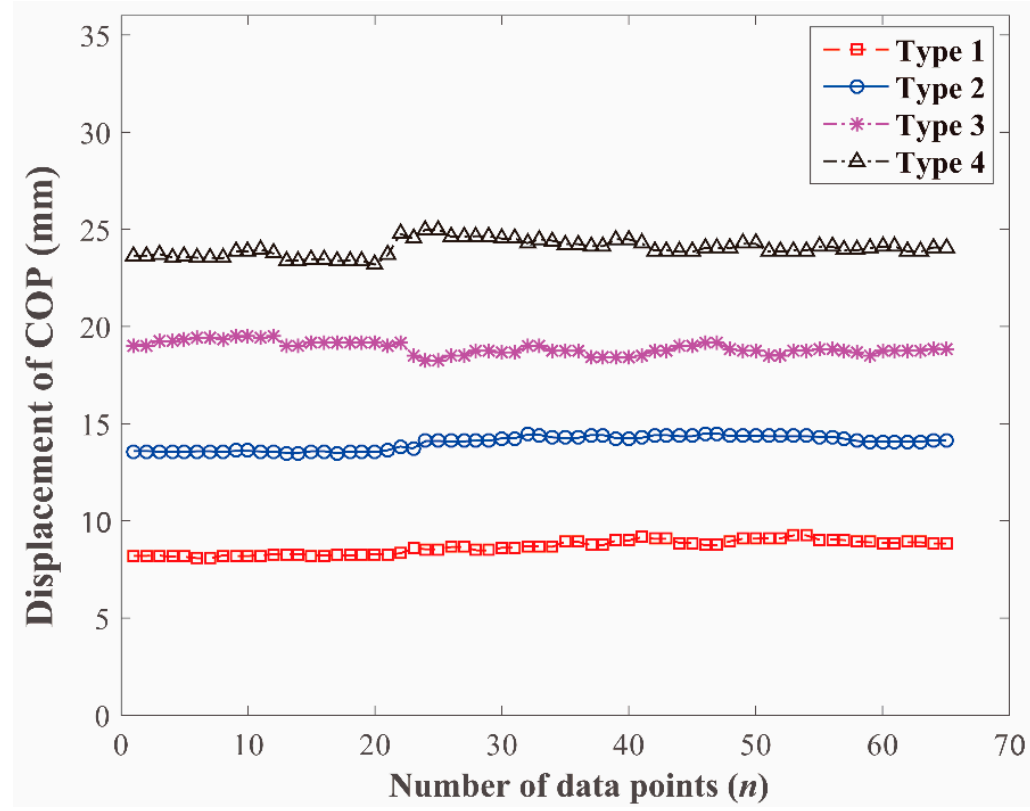

Figure 6. The representative displacement of COP for a subject sitting on a chair. As the number of spots used in the calculations increases, the displacement of COP decreases. The number of data points $(n)$ indicates the data collected at consecutive points in time.

\subsection{Walking}

Figure 7a shows the representative center-of-pressure trajectory of a subject during walking, and Figure $7 \mathrm{~b}$ presents the differences in the COP calculation of subject 2 by adopting different numbers of sensing spots. For the center-of-pressure trajectory shown in Figure $7 \mathrm{a}$, the trajectory started at the point $(17.7,153.2)$, and then the typical butterfly diagram appeared when the subject's walking speed stabilized after the acceleration. Among these four calculation forms, the RMSE values of Type 1 and Type 3 were all below $10 \mathrm{~mm}$ in both ML and AP directions (data shown in Table 3). Statistical results showed that a significant difference $(p<0.05)$ existed between Type 1 and Type 3 in the ML direction, yet there was no statistical difference between the RMSE values for Type 1 and Type 3 in the AP direction. Type 2 still had an inferior COP estimation in the AP direction, and Type 4 exhibited the worst results in the COP estimation. Statistical results showed that there were significant differences in RMSE values for Type 4 in the AP direction between Type 1 $(p<0.01)$, Type $2(p<0.05)$, and Type $3(p<0.01)$.

Table 3. The average and standard deviation values of RMSE for different calculation types during walking (values in table: mean \pm standard deviation; unit: $\mathrm{mm}$ ).

\begin{tabular}{ccccc}
\hline Types & Type 1 & Type 2 & Type 3 & Type 4 \\
\hline Direction & $3.6 \pm 0.3$ & $5.2 \pm 0.5$ & $7.1 \pm 2.1$ & $12.5 \pm 2.2$ \\
\hline ML direction & $5.3 \pm 2.0$ & $10.5 \pm 1.7$ & $8.4 \pm 4.1$ & $18.0 \pm 5.0$ \\
\hline
\end{tabular}




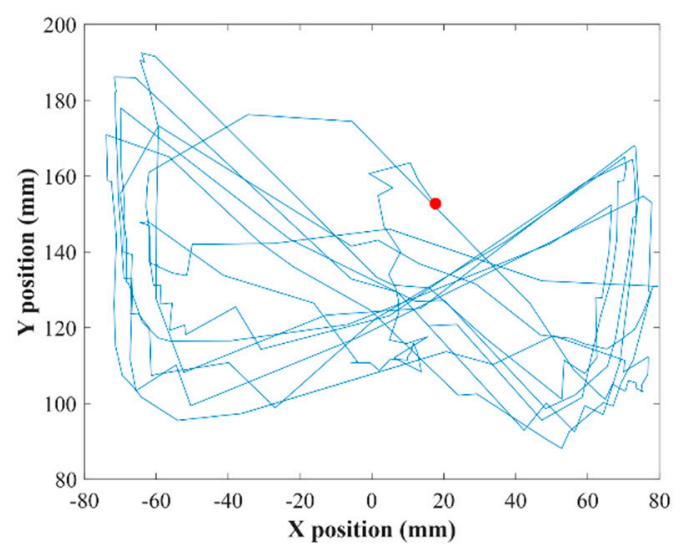

(a)

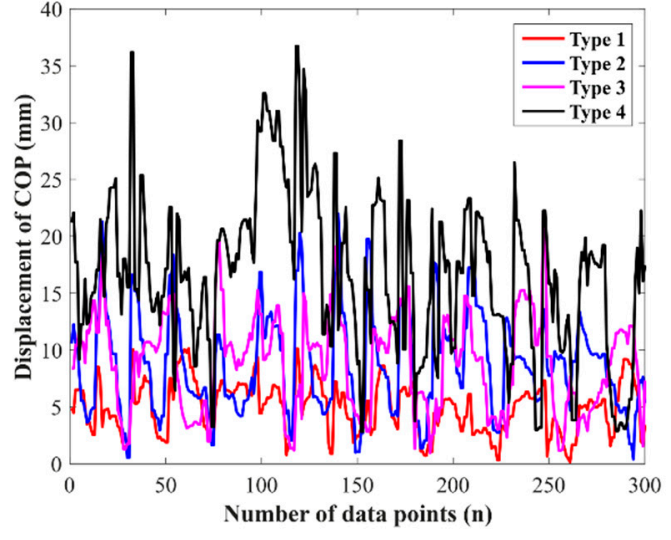

(b)

Figure 7. The trajectory and displacement of COP during walking. (a) The representative center-of-pressure trajectory of a subject during walking. The red circle indicate the starting point of trajectory of walking at $(17.7,153.2)$. (b) The differences in the COP calculation by adopting different numbers of sensing spots for subject 2 . The number of data points (n) indicates the data collected at consecutive points in time.

\subsection{COP Calcualtion Using Different Subregions of the Metatarsals}

By incorporating different subregions of the metatarsals into the calculations, the results reveal a noticeable finding, namely that the A combination (using the entire metatarsal region) during quiet standing did not exhibit the best result in the accuracy of the COP calculation (shown in Table 4). On the contrary, the B and D combinations, using fewer sensing data points, presented better results for the accuracy of the COP calculation. Among these four combinations, statistical results showed no significant differences between the RMSE values in the ML direction. Conversely, the $\mathrm{B}$ and $\mathrm{D}$ combinations presented better results in the accuracy of $\mathrm{COP}$ calculation in the AP direction compared with the $\mathrm{A}$ or $\mathrm{C}$ combinations (shown in Table 5). There wasno statistically significant difference between the $\mathrm{B}$ and $\mathrm{D}$ combinations. Table 6 shows the average and standard deviation values of RMSE for different combinations of metatarsal subregions during walking. The statistical analyses showed that there were no significant differences between the values of these four combinations in the ML direction. In the AP direction, the $\mathrm{D}$ combination reached a statistically significant level compared with the A/C combination $(p<0.05$; Table 7$)$. We conclude, therefore, that the locations of selected sensors indeed influence the accuracy of the COP calculation.

The B and D combinations both used 38 sensing spots in the COP calculation, and the number of spots is close to that of Type 1. Hence, statistical analysis was also utilized to compare the results of these three calculation modes while walking (results shown in Table 8). In the ML direction, Type 1 showed that there was a very significant difference $(p<0.01)$ with the B and D combinations. However, there was no statistically significant difference between these three calculation modes in the AP direction. In summary, Type 1 still provided the best result of COP approximation among the proposed calculation modes used in this study.

Table 4. The average and standard deviation values of RMSE for different combinations of metatarsal subregions during quiet standing (values in table: mean \pm standard deviation; unit: $\mathrm{mm}$ ).

\begin{tabular}{ccccc}
\hline Types & Type 1 & Type 2 & Type 3 & Type 4 \\
Direction & $2.8 \pm 0.9$ & $1.2 \pm 1.3$ & $2.9 \pm 2.6$ & $1.7 \pm 1.9$ \\
\hline ML direction & $11.5 \pm 1.4$ & $3.4 \pm 2.0$ & $11.0 \pm 1.6$ & $2.7 \pm 2.0$ \\
\hline AP direction &
\end{tabular}

(A) the entire metatarsal region; (B) medial and lateral metatarsal regions; (C) medial and middle metatarsal regions; (D) lateral and middle metatarsal regions. 
Table 5. ANOVA with post hoc Tukey test for the comparison of four combinations during quiet standing in the AP direction ( $p$ value).

\begin{tabular}{ccccc}
\hline Combinations & A & B & C & D \\
\hline A & - & $0.000^{* * *}$ & 0.958 & $0.000^{* * *}$ \\
B & & - & $0.000^{* * *}$ & 0.932 \\
C & & & - & $0.000^{* * *}$ \\
D & & & & - \\
\hline
\end{tabular}

$\overline{* * *}, p<0.001$ (very highly significant).

Table 6. The average and standard deviation values of RMSE for different combinations of metatarsal subregions during walking (values in table: mean \pm standard deviation; unit: $\mathrm{mm}$ ).

\begin{tabular}{ccccc}
\hline Combinations & Type 1 & Type 2 & Type 3 & Type 4 \\
\hline Direction & $6.1 \pm 2.7$ & $7.5 \pm 0.8$ & $6.1 \pm 2.0$ & $7.9 \pm 1.9$ \\
\hline ML direction & $11.5 \pm 2.6$ & $7.2 \pm 2.0$ & $11.7 \pm 2.7$ & $6.3 \pm 1.6$ \\
\hline
\end{tabular}

(A) the entire metatarsal region; (B) medial and lateral metatarsal regions; (C) medial and middle metatarsal regions; (D) lateral and middle metatarsal regions.

Table 7. ANOVA with post hoc Tukey test for the comparison of four combinations of walking in the AP direction ( $p$ value).

\begin{tabular}{ccccc}
\hline Combinations & A & B & C & D \\
\hline A & - & 0.076 & 1 & $0.029 *$ \\
B & & - & 0.067 & 0.94 \\
C & & & - & $0.025^{*}$ \\
D & & & & - \\
\hline$* p<0.05$ (significant). & & &
\end{tabular}

Table 8. ANOVA with post hoc Tukey test for the comparison of Type 1, B and D combinations of walking in the ML direction ( $p$ value).

\begin{tabular}{cccc}
\hline Types & Type 1 & B Combination & D Combination \\
\hline Type 1 & - & $0.004^{* *}$ & $0.002^{* *}$ \\
B combination & & & 0.886 \\
D combination & & & - \\
${ }^{* *}, p<0.01$ (highly significant). & &
\end{tabular}

In order to validate statistical results, we used the $G^{*}$ Power software to analyze the statistical power (for Tables 1-4 and Table 6). Experimental results with higher statistical power can decrease the probability of the false negative error. It can also serve to confirm the conclusions that can be drawn from the results of the designated study. All analysis results show that the values of statistical power are all greater than the minimum statistical power of 80\%, e.g., 0.80 (shown in Figures S1-S5).

\section{Discussion}

For the results obtained from quiet standing (Figure 5), we noticed that using fewer sensing spots for the COP calculation produced a larger deviation in the estimation of COP. A previous study found that the estimation accuracy of the foot plantar COP depended on the area coverage of pressure sensing spots on the insole [15], which also influenced the accuracy of the COP estimation. Our experiments presented the same result, and it was worth noting that Type 3 had nine fewer sensing points than Type 2; however, the results of COP estimation for Type 3 were better than for Type 2 and were close to the results obtained from Type 1. Initially, we expected that Type 2, with 29 spots for the COP calculation, would have small average RMSE values in the ML and AP directions, like Type 1. However, the results presented in the experiment were not as good as expected. 
We suggest that the reason for the larger RMSE value of Type 2 in the AP direction was the distribution of the selected spots. The distribution was also a key factor in influencing the accuracy of the COP trajectory estimation. During quiet standing, the RMSEs along the $\mathrm{AP}$ direction were greater than those along the ML direction for all calculation types. These consequences were reasonable because the COP trajectories usually had a greater movement range along the AP direction [15].

In our analysis of walking, the data from the insoles calculated by using Type 1 achieved the greatest accuracy of the COP trajectory estimation. It was worth noting that Type 2 used fewer sensing spots in the COP calculation, but it had slightly smaller RMSE values in the ML direction than Type 1 during quiet standing and sitting on a chair (Tables 1 and 2). We think the reason is that the foot pressure should be distributed evenly around the foot in the static condition, and Type 2 still can cover most areas of foot pressure at rest. During walking, the pressure varies quickly, and only by using more pressuresensing spots can more accurate COP estimation results be achieved. Compared with the original type, the number of spots used for the calculation could be reduced with the use of the Type 1 calculation form. For different applications in the IoHT, developers may consider manufacturing several types of smart insoles with varying degrees of accuracy and price. At present, many insoles with few pressure-sensing spots have been developed, and they are designed and applied in a pose-sensed virtual environment [16], gait analysis system for the IoHT [23], and a patient telemonitoring system [24].

The number of pressure-sensing cells was shown to influence the RMSE of the COP coordinates, which decreased as the number of cells increased from 2 to 7 [22]. The definition for subregions (cells) and the type of insole used in that study are different from ours. In our study, B, C, and D combinations all contain six subregions, and only the A combination has seven subregions.

Accurate calculation of results from pressure-sensing data is in high demand by both professional athletes and patients, and insoles with the pressure-sensing area covering the whole plantar region should be chosen. Edge computing can realize the distributed computing cloud infrastructure, which is also a very important key point for the development of 5G. By using this technique, it can create a better end-user experiment and bring other benefits, like low latency, high bandwidth, and security. The combination of smart insoles, distributed edge computation, and the cloud network is expected to produce more healthcare applications in the IoT infrastructure. Different types of smart insoles are being developed, some of which can measure the intact plantar pressure distributions while other low-cost insoles can just detect the pressure via the limited number of sensing spots distributed on the insoles.

By adopting 20 sensing spots in the calculation, Type 3 exhibits an acceptable result of COP approximation in the quiet standing and walking modes. It is only in the action of sitting that Type 3 has larger deviations of COP estimation in the AP and ML directions. The applications of smart insoles mainly focus on monitoring the activity of an individual, especially the analysis of plantar pressure and gait analysis; monitoring the static activity of sitting on a chair is not their main purpose. Hence, Type 3 is still suitable for use in the development of low-cost insoles for the consumer market. Even though the accuracy of Type 3 is not precise, it can meet the needs of the public and some healthcare cases that demand less accuracy for providing basic gait analysis and the basic measurement of running biomechanics.

Aside from the configurations of sensing points and the combinations we investigated in this study, it is worth noting that there are still many possibilities to generate other calculation configurations by using the same strategy. In these simplified calculation forms, it must not be considered obvious that a greater number of sensors necessarily gives a better result (Type 3 works better than Type 2), even when considering some reasonable criteria (evenly distribution on the foot-printed area) of the distribution of the selected sensing points. We suggest that other configurations of sensing points and combinations may also have chances to provide more representative values close to the true values of COP. 


\section{Conclusions}

This work presents a simplified calculation form that contains 36 sensing spots (Type 1) which can provide relatively accurate results for the COP calculations using the sensing data of insoles. This information is helpful for the design of the low-cost insole, and it is important to choose the appropriate smart insole according to individual needs. The location of selected sensors also demonstrates its importance in influencing the accuracy of the COP calculation in the analyses. According to the findings of this study, we recommend that the design of low-cost smart insoles must consider the distribution of the sensing spots, and the number of sensing spots also needs to be equal to or greater than 20. Healthy people can use the low-cost smart insoles to record daily steps, walking distance per day, and monitor their stability. For medical care applications, a higher accuracy of COP estimation is essential, and the pressure-sensing insoles that can sense all areas of foot pressure are to be preferred. The development of affordable low-cost insoles with the required degree of accuracy for applications can also allow the pressure-sensing insoles to be used in more fields. The studies of gait patterns of patients with the foot diseases, like hammertoes, hallux valgus, tailor's bunion or knee osteoarthritis, by using the smart insoles are our future research topics.

Supplementary Materials: The following are available online at https:/ /www.mdpi.com/2071-105 0/13/5/2934/s1, Figure S1: The examination of statistical power from the data shown in Table 1, Figure S2: The examination of statistical power from the data shown in Table 2, Figure S3: The examination of statistical power from the data shown in Table 3, Figure S4: The examination of statistical power from the data shown in Table 4, Figure S5: The examination of statistical power from the data shown in Table 6, Table S1: The specifications of the pressure sensing insole.

Author Contributions: W.-P.H. and L.-W.C. acquired the fund, conceived and designed the research; W.-P.H. analyzed and interpreted the data, and read drafts of the manuscript; J.-H.S. and H.-T.L. reviewed and edited; Y.-T.Y. helped perform the experiments. All authors have read and agreed to the published version of the manuscript.

Funding: This research was mainly funded by Asia University, Taiwan, under the contract number: ASIA-106-CMUH-26. This work was also supported in part by China Medical University Hospital (DMR-110-121), Asia University Hospital (10951025) and China Medical University's Higher Education SPROUT Project 2018-2022, MOE.

Institutional Review Board Statement: All participants were received and signed the informed consent form before experiments. The study was conducted according to the guidelines of the Declaration of Helsinki, and approved by the China Medical University \& Hospital Research Ethics Committee, Taichung City, Taiwan (CMUH107-REC2-018).

Informed Consent Statement: Informed consent was obtained from all subjects involved in the study.

Data Availability Statement: Data available on request due to restrictions e.g., privacy or ethical.

Acknowledgments: We acknowledge the technical support from the Industrial Technology Research Institute (Taiwan). Our gratitude also goes to Alison M. Fisher, professional English editor, for her assistance with proofreading.

Conflicts of Interest: The authors declare no conflict of interest.

\section{References}

1. Carek, A.M.; Conant, J.; Joshi, A.; Kang, H.; Inan, O.T. SeismoWatch: Wearable cuffless blood pressure monitoring using pulse transit time. Proc. ACM Interact. Mob. Wearable Ubiquitous Technol. 2017, 1, 40. [CrossRef] [PubMed]

2. Lersilp, S.; Putthinoi, S.; Lerttrakarnnon, P.; Silsupadol, P. Development and usability testing of an emergency alert device for elderly people and people with disabilities. Sci. World J. 2020, 2020, 5102849. [CrossRef]

3. Lin, F.; Wang, A.; Zhuang, Y.; Tomita, M.R.; Xu, W. Smart Insole: A Wearable Sensor Device for Unobtrusive Gait Monitoring in Daily Life. IEEE Trans. Ind. Inform. 2016, 12, 2281-2291. [CrossRef]

4. Olazarán, J.; Hernández-Tamames, J.A.; Molina, E.; García-Polo, P.; Dobato, J.L.; Álvarez-Linera, J.; Martínez-Martín, P. Clinical and anatomical correlates of gait dysfunction in Alzheimer's disease. J. Alzheimers Dis. 2013, 33, 495-505. [CrossRef] 
5. Verghese, J.; Lipton, R.B.; Hall, C.B.; Kuslansky, G.; Katz, M.J.; Buschke, H. Abnormality of gait as a predictor of non-Alzheimer's dementia. N. Engl. J. Med. 2002, 347, 1761-1768. [CrossRef] [PubMed]

6. Davie, C.A. A review of Parkinson's disease. Br. Med. Bull. 2008, 86, 109-127. [CrossRef]

7. Orlin, M.N.; McPoil, T.G. Plantar pressure assessment. Phys. Ther. 2000, 80, 399-409. [CrossRef]

8. Bacarin, T.A.; Sacco, I.C.N.; Hennig, E.M. Plantar pressure distribution patterns during gait in diabetic neuropathy patients with a history of foot ulcers. Clinics 2009, 64, 113-120. [CrossRef]

9. Saito, I.; Okada, K.; Nishi, T.; Wakasa, M.; Saito, A.; Sugawara, K.; Takahashi, Y.; Kinoshita, K. Foot pressure pattern and its correlation with knee range of motion limitations for individuals with medial knee osteoarthritis. Arch. Phys. Med. Rehabil. 2013, 94, 2502-2508. [CrossRef]

10. Saito, I.; Okada, K.; Wakasa, M.; Abe, H.; Saito, A. Foot pressure pattern, hindfoot deformities, and their associations with foot pain in individuals with advanced medial knee osteoarthritis. Gait Posture 2018, 59, 83-88. [CrossRef]

11. Vasconcelos, B.H.B.; Souza, G.S.; Barroso, T.G.C.P.; Silveira, L.C.L.; Sousa, R.C.M.; Callegari, B.; Xavier, M.B. Barefoot plantar pressure indicates progressive neurological damage in patients with human T-Cell lymphotropic virus type 1 infection. PLoS ONE 2016, 11, e0151855. [CrossRef]

12. Kalron, A.; Frid, L. The "butterfly diagram": A gait marker for neurological and cerebellar impairment in people with multiple sclerosis. J. Neurol. Sci. 2015, 358, 92-100. [CrossRef]

13. Nicolopoulos, C.S.; Anderson, E.G.; Solomonidis, S.E.; Giannoudis, P.V. Evaluation of the gait analysis FSCAN pressure system: Clinical tool or toy? Foot 2000, 10, 124-130. [CrossRef]

14. Godi, M.; Turcato, A.M.; Schieppati, M.; Nardone, A. Test-retest reliability of an insole plantar pressure system to assess gait along linear and curved trajectories. J. Neuroeng. Rehabil. 2014, 11, 95. [CrossRef]

15. Hu, X.; Zhao, J.; Peng, D.; Sun, Z.; Qu, X. Estimation of foot plantar center of pressure trajectories with low-cost instrumented insoles using an individual-specific nonlinear model. Sensors 2018, 18, 421. [CrossRef] [PubMed]

16. Lou, C.; Pang, C.; Jing, C.; Wang, S.; He, X.; Liu, X.; Huang, L.; Lin, F.; Liu, X.; Wang, H. Dynamic balance measurement and quantitative assessment using wearable plantar-pressure insoles in a pose-sensed virtual environment. Sensors 2018, 18, 4193. [CrossRef] [PubMed]

17. Tan, A.M.; Fuss, F.K.; Weizman, Y.; Woudstra, Y.; Troynikov, O. Design of low cost smart insole for real time measurement of plantar pressure. Procedia Technol. 2015, 20, 117-122. [CrossRef]

18. Weizman, Y.; Tan, A.M.; Fuss, F.K. Accuracy of centre of pressure gait measurements from two pressure-sensitive insoles. Proceedings 2018, 2, 277. [CrossRef]

19. Tao, J.; Dong, M.; Li, L.; Wang, C.; Li, J.; Liu, Y.; Bao, R.; Pan, C. Real-time pressure mapping smart insole system based on a controllable vertical pore dielectric layer. Microsyst. Nanoeng. 2020, 6, 62. [CrossRef]

20. Mach, P.; Becvar, Z. Mobile edge computing: A survey on architecture and computation offloading. IEEE Commun. Surv. Tutor. 2017, 19, 1628-1656. [CrossRef]

21. Coviello, G.; Avitabile, G. Multiple synchronized inertial measurement unit sensor boards platform for activity monitoring. IEEE Sens. J. 2020, 20, 8771-8777. [CrossRef]

22. Wang, D.; Cai, P.; Mao, Z. The configuration of plantar pressure sensing cells for wearable measurement of COP coordinates. Biomed. Eng. Online 2016, 15, 116. [CrossRef] [PubMed]

23. Dai, Y.; Xie, Y.; Chen, J.; Kang, S.; Xu, L.; Gao, S. A lamination-based piezoelectric insole gait analysis system for massive production for Internet-of-health things. Int. J. Distrib. Sens. Netw. 2020, 16. [CrossRef]

24. DeSilva, A.H.T.E.; Sampath, W.H.P.; Sameera, N.H.L.; Amarasinghe, Y.W.R.; Mitani, A. Development of a novel telecare system, integrated with plantar pressure measurement system. Inform. Med. Unlocked 2018, 12, 98-105. [CrossRef] 